\title{
Entlassung am nächsten Tag bei Einweisung nach Suizidankündigung - Pro
}

\author{
Discharge on the Day After Admission Following Announcement of Suicide - Pro
}

Pro

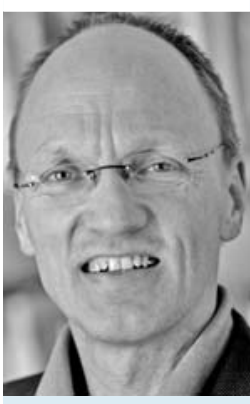

Tilman Steinert
Suizidankündigun-

gen haben sich phänomenologisch gewandelt. Als ich begann, in der Psychiatrie zu arbeiten, waren Suizidankündigungen ganz überwiegend ein Ereignis unter vier Augengegenüber dem Therapeuten oder gegenüber nahestehenden Personen. Auch Suizidankündigungen am Telefon haben einen Beziehungs- und Gesprächskontext und eine einfühlbare psychopathologische Färbung. Heute haben wir es inzwischen häufig mit einem anderen Phänomen zu tun: Die Suizidankündigung per SMS oder in sozialen Netzwerken, oft nur aus wenigen Worten bestehend und mit einem Inhalt, der alle Interpretationen offen lässt („macht euch keine Sorgen mehr um mich, danke für alles, was ihr für mich getan habt“). Mit Vorliebe an die beste Freundin oder den Exfreund, danach Mobiltelefon abgeschaltet, nicht mehr erreichbar, höchst erstaunt und vorwurfsvoll, wenn wenig später die Polizei vor der Türe steht. Im Gegensatz zur Vier-Augen-Mitteilung sind derartige SMS oder Facebook-Eintragungen kryptische Botschaften; aufgrund ihrer informativen Dürre gelingt es oft weder Angehörigen noch Professionellen, sie adäquat in einen diagnostischen bzw. psychopathologischen Kontext einzuordnen. Manchmal, aber keineswegs immer, stellt sich eine ernsthafte suizidale Gefährdung heraus, die einer Behandlung bedarf [1].

Die Aufgabe des Psychiaters und Psychotherapeuten ist es auch in diesen Fällen, Diagnostik und Differenzialdiagnostik vorzunehmen und gegebenenfalls eine angemessene Therapie einzuleiten. In der Differenzialdiagnostik von Suizidankündigungen gilt es im Wesentlichen, drei Typen zu unterscheiden.

\section{Patienten mit Suizidalität im Rahmen} einer Depression

Bei diesen ist die Suizidankündigung eindeutig ein Indikator für eine suizidale Gefährdung. Die Suizidalität ist im Wesentlichen durch kognitiv verzerrte depressive Fehlbewertungen bedingt. Diese Patienten bedürfen dringend einer Therapie, meistens stationär. In der Regel ist dafür das Einverständnis der Patienten zu gewinnen, ausnahmsweise ist auch einmal eine gerichtliche Unterbringung erforderlich. Ob tatsächlich ein depressives Syndrom vorliegt, lässt sich mit eingehender Exploration und Fremdanamnese sowie Verhaltensbeobachtungen auf der Station meistens innerhalb von 24 Stunden gut beurteilen.

2. Patienten mit Suizidankündigung unter Alkoholeinfluss

Alkohol ist bekanntermaßen in hohem Maße mit gewalttätigem Verhalten assoziiert, jedoch auch mit selbstgerichteter Aggressivität und Suizidalität. Angesichts der großen Häufigkeit, mit der dieses Phänomen klinisch beobachtet wird, ist die Behandlung in der Literatur eher spärlich; dennoch sind die $\mathrm{Zu}$ sammenhänge gut belegt [2, 3]. Nach klinischer Erfahrung sind nahezu alle Patienten, die in alkoholisiertem $\mathrm{Zu}$ stand Suizidgedanken oder -absichten äußern (mit oder ohne gleichzeitig bestehende Alkoholabhängigkeit) nach Ausnüchterung nicht mehr suizidal. Wenn keine weitergehende Indikation für eine stationäre Behandlung besteht, spricht meines Erachtens nichts gegen eine Entlassung nach Ausnüchterung und Beratung über Risiken und Behandlungsmöglichkeiten. Durchaus besteht die Gefahr der Wiederholung derartiger Ereignisse und mittelfristig ein erhöhtes Suizidrisiko [4].

3. Menschen mit Suizidankündigungen aus anderen Gründen ohne Vorliegen eines depressiven Syndroms

Gründe sind in der Regel psychosoziale Konfliktlagen, eventuell auch verbun- den mit manipulativen Intentionen bezüglich anderer Personen im persönlichen Umfeld, nicht selten (freilich keineswegs immer) vor dem Hintergrund einer Persönlichkeitsstörung. Gerade diese Fälle bedürfen einer sehr sorgfältigen Abklärung im Hinblick auf psychopathologische Symptomatik, belastende und protektive situative Faktoren und fortbestehende Suizidalität. Insbesondere muss ein hintergründig doch bestehendes schwereres depressives Syndrom sorgfältig eruiert beziehungsweise ausgeschlossen werden. Wenn ein depressives Syndrom nach hinreichend gründlicher diagnostischer Abklärung, die innerhalb von 24 Stunden gelingen sollte, nicht vorliegt und keine sonstige Indikation für eine stationäre Behandlung besteht, spricht meines Erachtens nichts gegen eine Entlassung, wiederum natürlich nach Beratung und Aufzeigen von Behandlungsoptionen. Es spricht aber sogar einiges gegen eine Fortsetzung der stationären Behandlung. Gerade bei Persönlichkeitsstörungen entsteht durch die von Selbstverantwortung entlastenden Rahmenbedingungen der stationären Behandlung häufig ein regressiver Sog, der zu weiterer repetitiver Symptomakzentuierung, einer sich selbst verstärkenden Spirale dann tatsächlich entstehender stationärer Behandlungsbedürftigkeit und somit einer schweren Destabilisierung des Gesundheitszustands führen kann [5]. Solche Patienten nach Suizidankündigungen womöglich noch gegen ihren Willen gerichtlich unterzubringen, könnte man bei sehr fraglichem Nutzen und möglichem erheblichem Schaden sogar für unethisch halten, wenngleich jeder klinische Psychiater Fälle kennt, in denen sich eine Negativspirale von Suizidankündigungen, Selbstverletzungen und Zwang nicht vermeiden ließ. Wer weder depressiv noch psychotisch, intoxikiert, delirant oder dement ist, ist auch in seinen Entscheidungen selbstbestimmt, ein- 
schließlich möglicher selbstschädigender Handlungen. Nach ausreichend gründlicher Abklärung der Sachlage und Anbieten möglicher Behandlungsoptionen hat der Arzt weder das Recht noch die Pflicht, solchen Patienten mit Zwang zu begegnen.

Unsere Behandlungsgewohnheiten im Hinblick auf die Indikationsstellung für stationäre Behandlungen folgen oft Traditionen und sind nicht sonderlich evidenzbasiert. In Deutschland gibt es einen breiten und kaum hinterfragten Konsens bei Psychiatern, anderen Ärzten, Polizei, Angehörigen und der Öffentlichkeit, dass Menschen mit Suizidäußerungen sofort in ein psychiatrisches Krankenhaus eingewiesen werden müssen. Ein Patient in England dagegen würde nach einer Suizidankündigung mit hoher Wahrscheinlichkeit noch am selben Tag einen Psychiater zu sehen bekommen, sehr wahrscheinlich aber kein psychiatrisches Krankenhausbett. Es gibt keinerlei Evidenz dafür, dass diese grundsätzlich andere klinische Praxis zu mehr Suiziden führt. Suizide stehen ganz überwiegend in Zusammenhang mit psychischen Erkrankungen, die Suizidrate ist in England deutlich niedriger als bei uns [6]. Nachdenklich stimmen die Ergebnisse einer kürzlich publizierten Studie über das Suizidrisiko im Zusammenhang mit psychiatrischer Be- handlung [7]. Es handelte sich um eine Analyse aller Suizide in Dänemark zwischen 1996 und 2009. Irgendeine Art psychiatrischer Behandlung erhöhte das Risiko um den Faktor 8; Patienten, die mit der Notaufnahme Kontakt gehabt hatten, hatten eine Risikoerhöhung um den Faktor 28 und solche, die stationär aufgenommen worden waren, hatte eine Risikoerhöhung um den Faktor 44. Mit Rückschlüssen bezüglich Ursachen ist große Vorsicht angebracht, Selektionsfaktoren spielen sicher eine Rolle. Trotzdem sind diese Daten nicht gerade ein Beleg für die Erfolge stationärer psychiatrischer Behandlung in der Suizidverhütung. Wir benötigen in diesem sehr wichtigen klinischen Tätigkeitsfeld eines Psychiaters mehr Evidenz. Gegebenenfalls sollten wir bereit sein, unsere klinischen Handlungsroutinen neuen Erkenntnissen anzupassen.

\section{Literatur}

1 Ruder TD, Hatch GM, Ampanozi G et al. Suicide announcement on Facebook. Crisis 2011; 32: 280-282

2 Borges G, Cherpital CJ, MacDonald S et al. A case-crossover study of acute alcohol use and suicide attempt. J Stud Alcohol Drugs 2004; 65: 708-714

3 Dawson DA. Alcohol, drugs, fighting and suicide attempt/ideation. Addict Res Theory 1997; 5: 451-472
4 Suokas J, Lönnqvist J. Suicide attempts in which alcohol is involved: a special group in general hospital emergency rooms. Acta Psychiatr Scand 1995; 91: 36-40

5 Steinert T, Steib L, Uhlmann C, Tschöke S. Stationäre Krisenintervention bei Patientinnen mit einer Borderline-Persönlichkeitsstörung. Göttingen: Hogrefe; 2014

6 Eurostat. National suicide rates. http://ec. europa.eu/eurostat/tgm/table.do?tab=table \&init $=1$ \&language $=e n \& p c o d e=t p s 00122 \&$ plugin $=1$

7 Hjorthøj CR, Madsen T, Agerbo E et al. Risk of suicide according to level of psychiatric treatment: a nationwide nested case-control study. Soc Psychiatry Psychiatr Epidemiol 2014; 49: 1357-1365

Sie haben eine eigene Meinung zu diesem

Thema? Dann schreiben Sie uns an:

psychiat-praxis@thieme.de!

Korrespondenzadresse

Prof. Dr. med. Tilman Steinert

Zentrum für Psychiatrie Süd-Württemberg, Weissenau, Abt. Psychiatrische Versorgungsforschung

Weingartshoferstraße 2

88214 Ravensburg-Weissenau

tilman.steinert@zfp-weissenau.de

Bibliografie

Dol http://dx.doi.org/

10.1055/s-0035-1552733

Psychiat Prax 2015; 42: 356-357

(c) Georg Thieme Verlag KG

Stuttgart · New York

ISSN 0303-4259 